
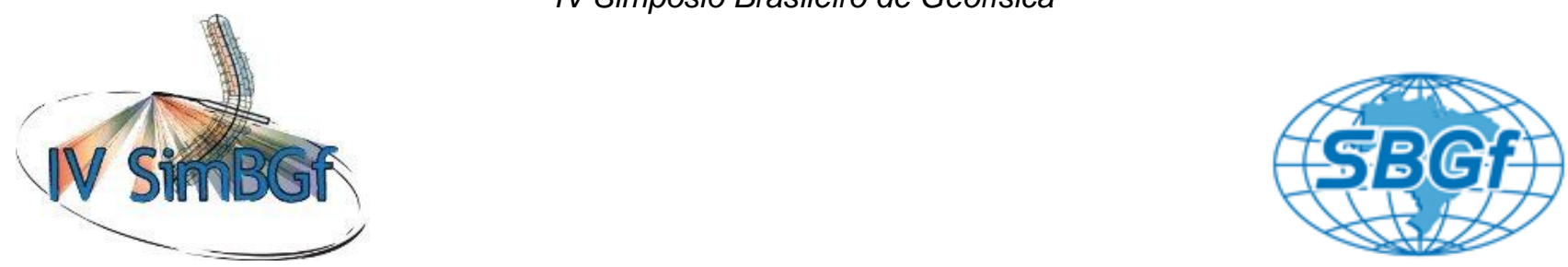

\title{
Parametrização de campo unidimensional de velocidades sísmicas usando ondaletas
}

Helcio Moreira Perin*, UFBA

Wilson Mouzer Figueiró, CPGG-UFBA

Copyright 2010, SBGf - Sociedade Brasileira de Geofísica

Este texto foi preparado para a apresentação no IV Simpósio Brasileiro de Geofísica, Brasília, 14 a 17 de novembro de 2010. Seu conteúdo foi revisado pelo Comitê Técnico do IV SimBGf, mas não necessariamente representa a opinião da SBGf ou de seus associados. É proibida a reprodução total ou parcial deste material para propósitos comerciais sem prévia autorização da SBGf.

\section{Abstract}

The aim of this work is the representation (parameterization) of compressional seismic velocity field model of stratified geological structure by means of orthogonal functions known as wavelets. More specifically, those so-called de Haar wavelet. Some comparative numerical experiments are performed among wavelet series with and without the use of scale function. The velocity field approximation, obtained by such technique, depends on the indices values used in the series, these have influence on the quality of the model representation. It is observed that the proposed parameterization offers a good accuracy for representation of the used field, and improves significantly when the scale function is considered in the wavelet series.

\section{Resumo}

O presente trabalho tem por objetivo a representação (parametrização) de modelo unidimensional de campo de velocidades sísmicas compressionais de uma estrutura geológica estratificada através da utilização de funções ortogonais conhecidas por ondaletas ou wavelets. Mais especificamente, aquelas denominadas ondaletas de Haar. São feitos experimentos comparativos entre as séries ondaleta com e sem o uso da função escala. A aproximação do campo de velocidade, obtida por essa técnica, depende dos valores de índices usados nas séries, estes influenciam a qualidade da representação do modelo. Observou-se que a parametrização proposta oferece uma boa acurácia na representação do campo utilizado, e melhora significativamente quando a função escala é considerada na série ondaleta.

\section{Introdução}

Alguns tipos de parametrização de campos de velocidades sísmicas são alvo de estudo por parte de alguns autores. Dos Santos e Figueiró (2006), definem dois deles: divisão do modelo em células ou blocos (tipo tradicional de parametrização usada na modelagem e inversão sísmicas, Bishop et al., 1985), e a polinomial trigonométrica (no qual o modelo é representado por uma única série trigonométrica).

O modelo de campo considerado é parametrizado pela ondaleta de Haar, que é definida como:

$\psi(t)=\left\{\begin{aligned} 1, & 0 \leq t<0,5 \\ -1, & 0,5 \leq t<1 \\ 0, & \text { caso contrário }\end{aligned}\right.$

que é também conhecida por ondaleta-mãe. Segundo Lima (2002), por definição, ondaleta é uma função $\psi(t) \in L^{1}(R) \cap L^{2}(R)$, tal que a família de funções

$\psi_{j, k}(t)=2^{j / 2} \psi\left(2^{j} t-k\right), j, k \in Z$

é uma base ortonormal para $L^{2}(R)$, espaço das funções quadrado integrável. As funções em (2) são conhecidas por ondaletas-filhas. Substituindo-se (2) em (1), temos:

$\psi_{j, k}(t)=\left\{\begin{array}{lc}2^{j / 2}, & 2^{-j} . k \leq t<2^{-j}(k+1 / 2) \\ -2^{j / 2}, & 2^{-j}(k+1 / 2) \leq t<2^{-j}(k+1) \\ 0, & \text { caso contrário }\end{array}\right.$

A expressão (3) foi obtida a partir da ondaleta de Haar, por uma dilatação binária $2^{j}$ e uma translação diádica $k 2^{-j}$ (Morenttin, 2004).

Pode-se, então, representar uma função tal como:

$f(t)=\sum_{j \geq j_{O}}^{+\infty} \sum_{k=-\infty}^{+\infty} d_{j, k} \psi_{j, k}(t)$

Onde:

$d_{j, k}=<f, \psi_{j, k}>=\int_{-\infty}^{+\infty} f(t) \psi_{j, k}(t) d t$

Um fator de grande importância para a obtenção de uma boa aproximação, é a introdução da função escala (ondaleta-pai), que é representada por $\phi_{j o, k}(t)$. A função escala de Haar é: $\phi(t)= \begin{cases}1, & 0 \leq t \leq 1 \\ 0, & \text { caso contrário }\end{cases}$

Tal como em (3), temos:

$\phi_{j o, k}(t)=2^{j o / 2}, \quad 2^{-j o} \cdot k \leq t \leq 2^{-j o} .(k+1)$

Considerando um sistema ortonormal das funções $\psi(t)$ e $\phi(t)$, pode-se fazer uma aproximação de $\mathrm{f}(\mathrm{t})$, como segue:

$f(t)=\sum_{k=-\infty}^{+\infty} c_{j_{o}, k} \phi_{j_{o}, k}(t)+\sum_{j \geq j_{o}}^{+\infty} \sum_{k=-\infty}^{+\infty} d_{j, k} \psi_{j, k}(t)$

Onde: 


$$
c_{j_{o}, k}=<f, \phi_{j o, k}>=\int_{-\infty}^{+\infty} f(t) \phi_{j_{o}, k}(t) d t
$$

Para finalidades práticas, os somatórios que aparecem em (4) e (7) devem ser finitos. Portanto, deve-se escolher um conjunto de valores inteiros dentro do qual $j$ e $k$ variam. A aproximação de $\mathrm{f}(\mathrm{t})$ por ondaletas depende fortemente do número de coeficientes $j$ e $k$ utilizados (inteiros e arbitrários); quanto maior o intervalo de valores utilizados para $j$ e $k$, melhor será a aproximação. O índice jo é denominado escala de resolução e deve ser igual ou maior ao valor inicial de $j$ escolhido.

\section{Aplicação da Metodologia}

Consideremos um modelo geológico estratificado, constituído de cinco camadas horizontais (Figura 01). Trata-se de um modelo em que a velocidade compressional $\mathrm{V}$ depende apenas da profundidade $\mathrm{Z}$.

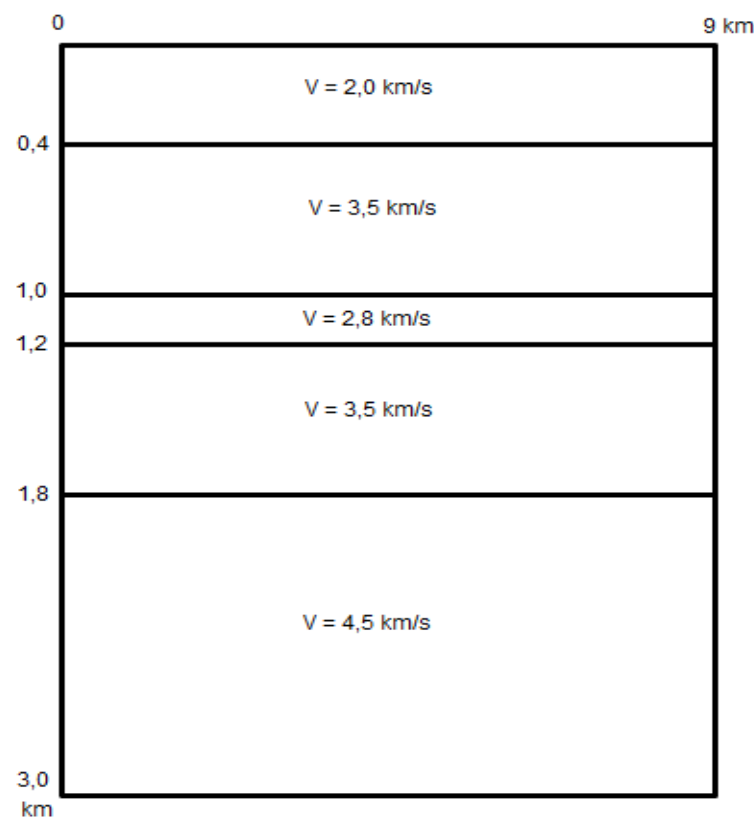

Figura 01 - Velocidade compressional de modelo geológico de camadas com interfaces horizontais.

A função $V(z)$ que representa esse modelo de 5 camadas é dado, em km/s, por

$$
V(z)= \begin{cases}2,0 & 0 \leq z<0,4 \\ 3,5 & 0,4 \leq z<1 \\ 2,8 & 1 \leq z<1,2 \\ 3,5 & 1,2 \leq z<1,8 \\ 4,5 & 1,8 \leq z<3\end{cases}
$$

Utilizamos as expressões (3), (4), (5), (6), (7) e (8) para representar tal modelo por série ondaleta de Haar, sendo $\mathrm{f}(\mathrm{t})$ substituído por $\mathrm{V}(\mathrm{z})$.

Inicialmente, utilizou-se como metodologia o cálculo manual dessas expressões. Devido à grande quantidade de cálculo, principalmente dos coeficientes $d_{i, k}$ e $c_{j o, k}$, fezse necessário a utilização de programa de computador. Foi desenvolvido um programa na linguagem FORTRAN a fim de suprir essa necessidade. Os valores dos índices $j, k$ e jo, foram dados de entrada do programa, sendo valores inteiros e arbitrários. Optou-se por utilizar valores iniciais e finais de $j$ e $k$ iguais em valor absoluto, por facilidade de interpretação. Foram testados vários valores de entrada para esses índices e escolhidos três exemplos que contemplam de forma resumida as várias situações possíveis. A partir dos dados de saída obtidos na execução do programa, fez-se os gráficos (Figuras 02, 03 e 04) que ilustram os resultados obtidos nos experimentos numéricos realizados.

A aproximação foi feita ponto a ponto, com espaçamento em z de $0,01 \mathrm{~km}$. Nos gráficos foi utilizado um espaçamento de $0,05 \mathrm{~km}$.

\section{Resultados}

Fazendo-se a parametrização através da ondaleta de Haar conforme a metodologia descrita, apresentamos, a seguir, alguns exemplos desenvolvidos a partir de situações distintas.

Exemplo 01 - Fazendo os cálculos manualmente, foi escolhido um baixo número de coeficientes; optou-se por fazer variações de -1 a 1 , tanto para valores de $j$ quanto para valores de $k$, referentes à ondaleta mãe. Para a primeira parte da série, referente à ondaleta pai, foi utilizado jo igual ao valor inicial de $j$, ou seja, -1 . Nesse caso, foi necessário o cálculo de nove coeficientes $d_{j, k} e$ três coeficientes $c_{j o, k}$. Com esses valores a resolução fica prejudicada na medida em que foram utilizados poucos coeficientes. A Figura 02 ilustra o Exemplo 1 sem a influência da função escala considerou-se apenas $\psi(z)$ e com influência da mesma considerou-se $\psi(z)$ e $\phi(z)$ para jo igual a -1 .

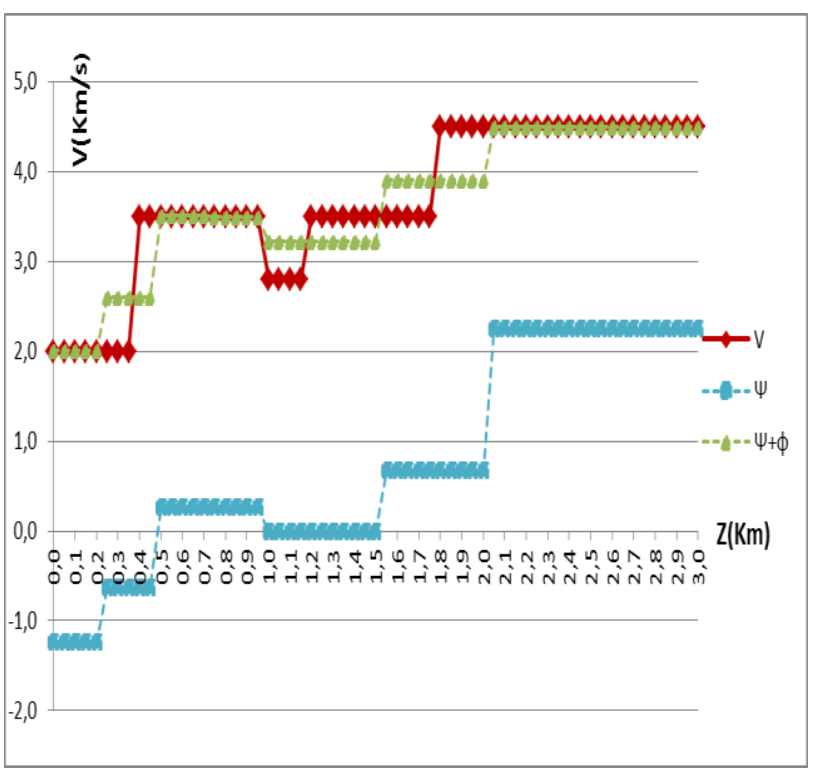

Figura 02 - Modelo $V(z)$ parametrizado por ondaleta de Haar sem e com a consideração de $\phi(z)$ para $-1 \leq \mathrm{j} \leq 1,-1 \leq \mathrm{k} \leq 1, \mathrm{jo}=-1, \mathrm{j}, \mathrm{k}$ e jo $\in \mathrm{Z}$. 
Diante das limitações envolvidas no cálculo manual, fezse necessário a utilização de um programa de computador com o objetivo de processar esses cálculos para essa ou qualquer outra função unidimensional. Assim, foram testados vários valores para $j, k$ e jo. Tal procedimento permitiu a análise da influência desses índices, bem como da função escala.

Os exemplos seguintes foram computacionalmente obtidos.

Exemplo 02 - O gráfico a seguir (Figura 03) mostra a estrutura geológica representada, utilizando-se $j$ variando de -5 a $5, k$ variando de -5 a 5 e jo igual a -5 (cálculo de 132 coeficientes).

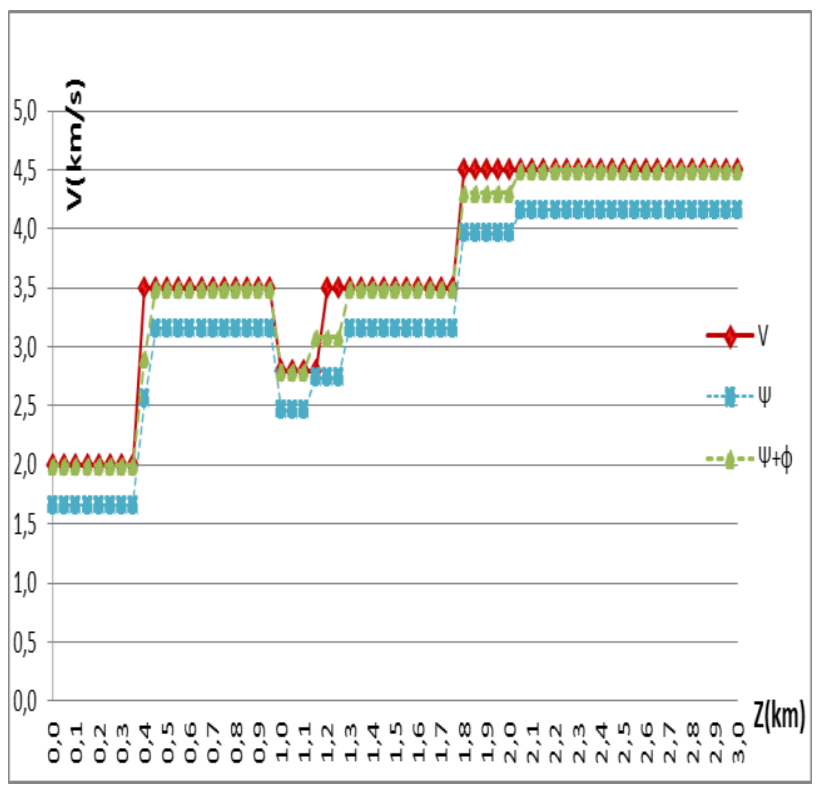

Figura 03 - Modelo V(z) parametrizado por ondaleta de Haar sem e com a consideração de $\phi(z)$ para $-5 \leq \mathrm{j} \leq 5,-5 \leq \mathrm{k} \leq 5$, Jo $=-5, \mathrm{j}, \mathrm{ke} \mathrm{jo} \in \mathrm{Z}$.

Comparado com o exemplo anterior, percebe-se uma diferença menor entre as séries com e sem a função escala. Ainda assim essa diferença é bastante significativa, sendo imprescindível para uma boa aproximação utilizando-se essa variação dos índices $j$ e $k$.

Exemplo 03 - Considerando-se $j$ variando de -15 a $15, k$ variando de -15 a 15 e jo igual a -14, obtém-se os resultados ilustrados no gráfico mostrado na Figura 04. Nesse caso, são calculados 992 coeficientes.

Observa-se que as curvas relativas aos casos sem e com utilização de $\phi(z)$ são praticamente coincidentes, sendo que a diferença entre elas é da ordem de 0.001, imperceptível no gráfico. Nesse nível de resolução, para esse exemplo, a aproximação é quase perfeita, diferindo apenas em quatro pontos. A partir desse nível, a razão entre custo computacional e a resolução torna-se elevada, ou seja, observa-se pouca melhora na resolução com grande aumento no número de coeficientes.

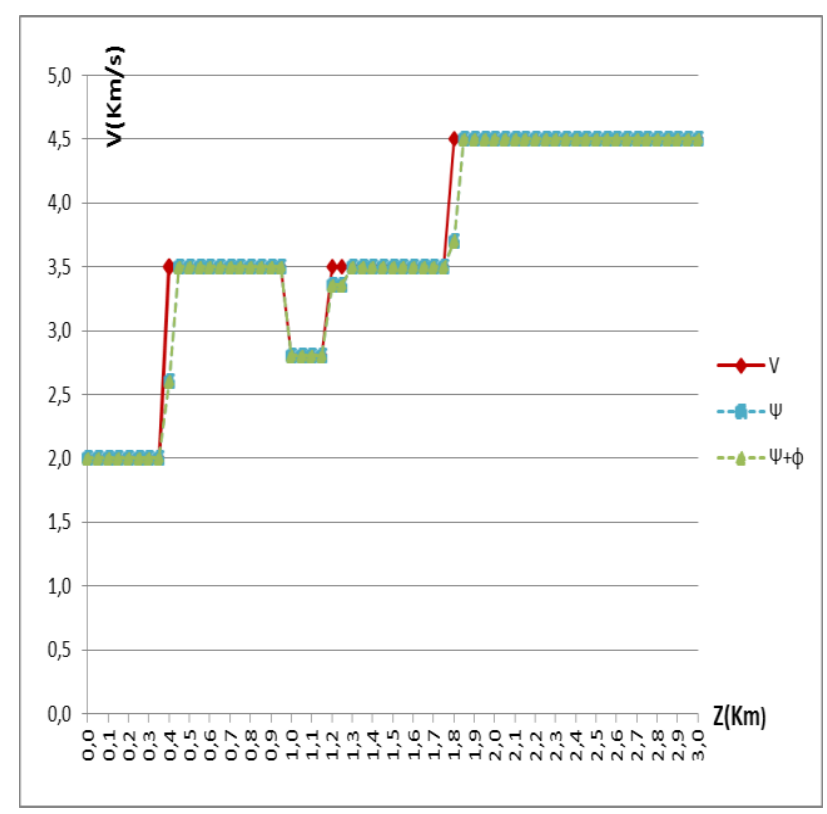

Figura 04 - Modelo $\mathrm{V}(\mathrm{z})$ parametrizado por ondaleta de Haar sem e com a consideração de $\phi(z)$ para $-15 \leq \mathrm{j} \leq 15,-15 \leq \mathrm{k} \leq 15, \mathrm{jo}=-14, \mathrm{j}, \mathrm{k}$ e jo $\in \mathrm{Z}$.

\section{Discussão e Conclusões}

Substituindo-se valores de $z$ na equação acima, observou-se uma discrepância em relação à $\mathrm{V}(\mathrm{z})$, principalmente no início da função. Até certo ponto essa diferença era esperada, visto que utilizou-se um intervalo pequeno para valores de $j$ e $k$. $O$ aumento desse intervalo implicaria em um maior esforço no cálculo, que foi feito manualmente.

No intuito de aperfeiçoar o método, fez-se necessário a implementação de um programa de computador que execute os cálculos. Dessa forma, pôde-se testar diferentes intervalos para valores de $j$ e $k$, diferentes valores de $j o$, etc., de forma a se obter resultados satisfatórios.

Para baixas resoluções (Exemplos 1 e 2), percebeu-se que a influência da função escala é muito grande, fazendo com que a aproximação seja razoável. Nesses casos, a melhor escolha para o jo é fazê-lo igual ao valor inicial de $j$.

Para resoluções mais elevadas (Exemplo 3), percebeu-se que a influência da função escala é mínima, ou seja, não melhora significativamente a aproximação. Nesses casos pode-se utilizar um jo com valor superior ao valor inicial de $j$.

A vantagem de se utilizar a função escala da ondaleta, reside no fato de que para se obter aproximações equivalentes, o uso dessa função exige um número de 
cálculos significativamente inferior ou um custo computacional menor.

Os objetivos foram alcançados, visto que a parametrização de um modelo geológico por ondaleta de Haar mostrou-se eficaz. No exemplo 03, por exemplo, em alguns segundos de processamento obteve-se uma aproximação quase que perfeita. Além disso, pôde-se analisar com clareza os índices $j, k$ e jo necessários para uma boa aproximação, para esse tipo de modelo.

Em se tratando de modelos mais complexos, é de se esperar a necessidade de maiores valores para $j$ e $k$. A partir desse ponto, pretende-se desenvolver técnicas, através do uso de funções auxiliares, no intuito de cobrir problemas mais complexos em duas dimensões. Pretende-se, também fazer parametrização de modelos geológicos por ondaleta de Haar, a partir de dados discretizados, obtidos experimentalmente.

\section{Referências}

Bishop, T.N. et al. (08 co-authors), 1985. Tomographic determination of velocity and depth in laterally varying media. Geophisics, 50: 903-923.

Morenttin, P. A., 2004. Ondas e ondaletas; da análise de Fourier à análise de ondaletas. EDUSP.

Lima, P. C., 2002. Wavelets: uma introdução. Matemática Universitária, $\mathrm{N}^{0}$. 33: 13-44.

Santos, R. H. M. \& Figueiró, W. M., 2006. Modelagem acústica bidimensional usando diferentes parametrizações de campos de velocidades. Revista Brasileira de Geofísica. Vol. 24(1): 103-115.

\section{Agradecimentos}

À Universidade do Estado da Bahia (UNEB), pela liberação para realização do curso de doutorado.

Ao Centro de Pesquisa em Geofísica e Geologia (CPGG / UFBA), pelo suporte técnico, logístico e computacional.

À Fundação de Amparo à Pesquisa do Estado da Bahia (FAPESB), pelo apoio financeiro através de bolsa de doutorado. 